\title{
Estudo epidemiológico de crianças com condiloma acuminado em um ambulatório de especialidades médicas na cidade de São Paulo
}

\author{
Epidemiological study of children with condyloma acuminata in an outpatient clinic of medical \\ specialties in the city of São Paulo \\ Estudio epidemiológico de niños con condiloma acuminado en un ambulatorio de especialidades \\ médicas de la ciudad de São Paulo
}

\begin{abstract}
Resumo
Tecnicamente, as verrugas genitais são denominadas como Condiloma Acuminado e popularmente são chamadas de "crista de galo". São lesões cutâneas causadas pelo vírus HPV, podendo surgir em diversos pontos da pele do paciente. O objetivo principal deste trabalho pautou-se em avaliar a epidemiologia de crianças com Condiloma Acuminado por meio da descrição das formas de transmissão do condiloma em crianças e das características clínicas e os tratamentos realizados para infecção pelo papilomavírus. Foi realizado um estudo retrospectivo dos prontuários, utilizando dados colhidos de prontuários eletrônicos dos pacientes com Condiloma Acuminado. A análise buscou dados como: sexo, idade, manifestação clínica, sítio da lesão, quantidade de lesão, possível modo de transmissão, tipo de parto, presença de lesão nas mães, tratamento e quantidade de sessões do tratamento. Foram avaliados 1816 portuários de pacientes submetidas a procedimento cirúrgico ginecológico no Ambulatório Médico Especializado Barradas (AME BARRADAS), no período compreendido entre os anos de 2013 a 2017. Para a composição da amostra deste estudo foram selecionados os pacientes que realizaram o tratamento no período estudado e atenderam aos critérios de elegibilidade: Crianças portadoras de Condiloma Acuminado Anogenital. Vale ressaltar que foram respeitados todos os aspectos contidos na Resolução 196/96. Os resultados apontaram que, com relação ao sexo, o condiloma é mais frequente em meninas e é mais provável que esteja associado a transmissão vertical na maioria dos casos (56,5\%). Trabalhos futuros são necessários para que se possa avaliar a relação da doença com a via de parto.
\end{abstract}

Palavras-chave: Papilomavírus Humano; Infecções por Papillomavirus; Verrugas genitais.

\begin{abstract}
Technically, genital warts are called Condyloma Acuminatum and are popularly called "cockscomb". They are skin lesions caused by the HPV virus, which can appear in different points of the patient's skin. The main objective of this work was to evaluate the epidemiology of children with Condyloma Acuminatum through the description of the forms of transmission of condyloma in children and the clinical characteristics and treatments performed for papillomavirus infection. A retrospective study of medical records was performed, using data collected from electronic medical records of patients with Condyloma Acuminatum. The analysis sought data such as: sex, age, clinical manifestation, lesion site, amount of lesion, possible mode of transmission, type of delivery, presence of lesion in mothers, treatment and number of treatment sessions. A total of 1816 port patients who underwent gynecological surgery at the Barradas Specialized Medical Outpatient Clinic (AME - BARRADAS) were evaluated in the period between 2013 and 2017. For the composition of the sample of this study, patients who underwent treatment in the period were selected. studied and met the eligibility criteria: Children with anogenital condyloma acuminatum. It is worth mentioning that all aspects contained in Resolution 196/96 were respected. The results showed that, in terms of gender, condyloma is more frequent in girls and is more likely to be associated with vertical transmission in most cases (56.5\%). Future work is needed in order to assess the relationship between the disease and the mode of delivery.
\end{abstract}

Keywords: Human Papillomavirus; Papillomavirus infections; Genital warts. 


\begin{abstract}
Resumen
Técnicamente, las verrugas genitales se denominan Condiloma Acuminado y popularmente se denominan "cresta de gallo". Son lesiones cutáneas provocadas por el virus VPH, que pueden aparecer en diferentes puntos de la piel del paciente. El objetivo principal de este trabajo fue evaluar la epidemiología de los niños con Condiloma Acuminado a través de la descripción de las formas de transmisión del condiloma en niños y las características clínicas y tratamientos realizados para la infección por papilomavirus. Se realizó un estudio retrospectivo de historias clínicas, utilizando datos recogidos de historias clínicas electrónicas de pacientes con Condiloma Acuminado. El análisis buscó datos como: sexo, edad, manifestación clínica, sitio de la lesión, cuantía de la lesión, posible modo de transmisión, tipo de parto, presencia de lesión en las madres, tratamiento y número de sesiones de tratamiento. Se evaluaron un total de 1816 pacientes portuarias que se sometieron a cirugía ginecológica en el Consultorio Médico Especializado Ambulatorio de Barradas (AME - BARRADAS) en el período comprendido entre los años 2013 y 2017. Para la composición de la muestra de este estudio se consideraron las pacientes que se sometieron a tratamiento en el período. seleccionados, estudiados y que cumplieron con los criterios de elegibilidad: Niños con Condiloma Acuminado Anogenital. Cabe mencionar que se respetaron todos los aspectos contenidos en la Resolución 196/96. Los resultados mostraron que, en cuanto al género, el condiloma es más frecuente en niñas y es más probable que se asocie a transmisión vertical en la mayoría de los casos $(56,5 \%)$. Se necesita trabajo futuro para evaluar la relación entre la enfermedad y el modo de parto.
\end{abstract}

Palabras clave: Virus del Papiloma Humano; Infecciones por Virus del Papiloma; Verrugas genitales.

\title{
1. Introdução
}

As verrugas anogenitais resultam da infecção pelo papiloma vírus humano (HPV). O HPV pode infectar tanto pele como mucosas. A maioria das infecções regride espontaneamente dentro de dois anos em pessoas com imunidade preservada. Mais de 130 subtipos de HPV são identificados até o momento, embora apenas cerca de um terço deles possa infectar a mucosa anogenital, oral, ou laríngea, predominando os subtipos 6, 11 e 16 (Cherry et al., 2013).

A transmissão das verrugas anogenitais induzidas pelo vírus HPV podem ocorrer nas crianças como transmissão vertical, inoculação digital ou por meio de outras lesões, fômites por diferentes modalidades de abuso sexual, seja pelo contato oral-genital, genital-genital, genital-anal, ou manipulação digital da vagina e/ou do ânus da criança (Armstrong \& Handley, 1997; Syrjanen \& Puranen, 2000).

A interpretação de condiloma anogenital em crianças como prova de abuso sexual é controversa, visto que a prevalência do HPV em crianças abusadas sexualmente varia de 5\% a 33\% e em crianças sem suspeita de abuso ficam em torno de $16 \%$ (Unger et al., 2011).

A manifestação clínica mais frequente é a presença de verrugas em sua maioria assintomáticas, sendo um achado casual durante troca de fraldas, banhos ou evidenciado pelo pediatra durante o exame físico (Emans et al., 2005).

O diagnóstico de uma doença sexualmente transmissível (DST) na criança corrobora com a queixa do abuso sexual, ou motiva sua investigação quando não existe tal alegação (Hammerschlag \& Guillén, 2010; Siegfried et al., 1998). Alguns autores consideram o diagnóstico de uma DST na infância suficiente para requerer avaliação que exclua o abuso sexual (Bechtel, 2010). A maioria dos estudos concorda, no entanto, que a presença isolada de uma DST é insuficiente para assegurar sua ocorrência (Hammerschlag \& Guillén, 2010).

O tratamento das lesões decorrentes do HPV deve ser individualizado. Podem ser necessárias várias sessões de modalidades terapêuticas distintas para resolução completa das lesões. O tratamento convencional consiste na destruição química da lesão com aplicação tópica de podofilina, 5-fluorouracil, ácido bi/tricloroacético e podofilotoxina. Os métodos de destruição física incluem crioterapia, laser, eletrocauterização e excisão cirúrgica. Como imunoterápicos podemos citar: interferon e imiquimode 5\% creme (Moresi et al., 2001).

Considerando o aumento da incidência do condiloma acuminado em crianças, observa-se a importância da realização desse estudo para realização de uma melhor classificação epidemiológica. 
Por conta disso, o objetivo principal deste trabalho pautou-se em avaliar a epidemiologia de crianças com Condiloma Acuminado por meio da descrição das formas de transmissão do condiloma em crianças e das características clínicas e os tratamentos realizados para infecção pelo papilomavírus.

\section{Material e Métodos}

\subsection{Material}

Foram avaliados 1816 prontuários de pacientes submetidos a procedimento cirúrgico ginecológico no Ambulatório Médico Especializado Barradas (AME - BARRADAS), no período entre os anos de 2013 a 2017. Para a composição da amostra deste estudo foram selecionados os pacientes que realizaram o tratamento no período estudado e atenderam aos critérios de elegibilidade: Crianças portadoras de condiloma acuminado anogenital.

Os critérios de exclusão foram: adolescentes e adultos que fizeram tratamento cirúrgico ginecológico. Desta forma, constituíram a amostra deste estudo, prontuários de 23 indivíduos que foram atendidos pelo setor e que haviam sido submetidos a tratamento.

Para levantamento dos aspectos epidemiológicos e clínicos dos pacientes com condiloma acuminado foi utilizado o computador do ambulatório de ginecologia do AME - BARRADAS para acesso integral aos prontuários eletrônicos dos respectivos pacientes.

\subsection{Método Clínico}

Foi realizado um estudo retrospectivo dos prontuários, utilizando dados colhidos dos prontuários eletrônicos dos pacientes com condiloma acuminado. A análise buscou dados como: sexo, idade, manifestação clínica, sítio da lesão, quantidade de lesão, possível modo de transmissão, tipo de parto, presença de lesão nas mães, tratamento e quantidade de sessões do tratamento. Os dados foram organizados em tabela para facilitar correlação e análise estatística.

As informações coletadas tiveram a garantia do sigilo que assegura a privacidade e o anonimato dos sujeitos quanto aos dados confidenciais envolvidos na pesquisa.

Foram respeitados todos os aspectos contidos na Resolução 196/96.

Esta pesquisa contou com a aprovação do Comitê de Ética em Pesquisa da instituição, processo no 79623317.6.0000.0083.

A análise estatística dos dados foi realizada com auxílio do programa Excel para elaboração dos gráficos e tabelas, e o resultado foi confrontado com a literatura pertinente.

\section{Resultados}

O sexo predominante nos pacientes foi o feminino, com $82,6 \%$ de participação. (Tabela 1).

Tabela 1. Distribuição dos casos segundo sexo do paciente.

$\begin{array}{ccc}\text { Sexo } & \mathbf{N}^{\mathbf{0}} \text { de Casos } & \% \\ \text { F } & 19 & 82,6 \% \\ \text { M } & 4 & 17,4 \% \\ \text { Total } & \mathbf{2 3} & \mathbf{1 0 0 , 0 \%}\end{array}$

Fonte: Autores. 
A idade das pacientes variou de 1 a 10 anos, com média de 4 anos e 8 meses, dos quais 43,5\% tinha entre 1 e 3 anos e $34,8 \%$ entre 4 e 6 anos (Tabela 2).

Tabela 2. Condiloma acuminado em crianças. Distribuição dos casos segundo a faixa etária.

$\begin{array}{cccc} & \text { Idade } & \mathbf{N}^{\mathbf{0}} \text { de Casos } & \text { \% } \\ & 0-3 & 10 & 43,5 \% \\ & 4-6 & 8 & 34,8 \% \\ \text { Total } & 7-10 & 5 & 21,7 \% \\ \end{array}$

Fonte: Autores.

A manifestação clínica predominante foi a presença de verrugas, encontradas em $100 \%$ dos pacientes (Tabela 3). A quantidade de lesões variou de 2 a 8 lesões (Tabela 4), Em 39,1\% dos casos as lesões ocorreram na região perianal. Localizações isoladas em região vulvo-perianal e vulva foram observadas em 34,8\% e 26,1\% dos casos respectivamente (Tabela 5).

Tabela 3. Manifestação clínica (queixa e achados) em crianças portadoras de condiloma acuminado.

\begin{tabular}{ccc} 
Manifestação Clínica & $\mathbf{N}^{\mathbf{0}}$ de Casos & $\mathbf{\%}$ \\
\hline VERRUGAS & 23 & $100,0 \%$ \\
Total & $\mathbf{2 3}$ & $\mathbf{1 0 0 , 0 \%}$
\end{tabular}

Fonte: Autores.

Tabela 4. Distribuição dos casos de condiloma acuminado em crianças segundo a quantidade de lesão.

$\begin{array}{|ccc|}\text { Quantidade de Lesão } & \mathbf{N}^{\mathbf{0}} \text { de Casos } & \text { \% } \\ 0-3 & 8 & 34,8 \% \\ 4-6 & 9 & 39,1 \% \\ 7-10 & 6 & 26,1 \% \\ \text { Total } & \mathbf{2 3} & \mathbf{1 0 0 , 0 \%}\end{array}$

Fonte: Autores.

Tabela 5. Distribuição dos casos de condiloma acuminado em crianças segundo sítio da lesão.

$\begin{array}{ccc}\text { Sitios da Lesão } & \mathbf{N}^{\mathbf{0}} \text { de Casos } & \mathbf{\%} \\ \text { PERIANAL } & 9 & 39,1 \% \\ \text { VULVAR } & 6 & 26,1 \% \\ \text { VULVAR / PERIANAL } & 8 & 34,8 \% \\ \text { Total } & \mathbf{2 3} & \mathbf{1 0 0 , 0 \%}\end{array}$

Fonte: Autores.

Para determinar a fonte de infecção, utilizamos levantamento de prontuários. Em 65,2\% tivemos a transmissão vertical como provável fonte de infecção, em 8,7\% dos prontuários foram registrados abuso sexual (Figura 6). Partos normais representaram 56,5\% dos tipos de partos dos pacientes (Figura 7). Foram detectados que 12 mães apresentavam lesões verrugosas, ou seja em 52,2\% dos casos (Tabela 8). 
Tabela 6. Fontes de Infecção pelo papilomavírus.

$\begin{array}{ccc}\text { Provável Fonte de Infecção } & \mathbf{N}^{\mathbf{0}} \text { de Casos } & \text { \% } \\ \text { VERTICAL } & 15 & 65,2 \% \\ \text { SEXUAL } & 2 & 8,7 \% \\ \text { DESCONHECIDO } & 6 & 26,1 \% \\ \text { Total } & \mathbf{2 3} & \mathbf{1 0 0 , 0 \%}\end{array}$

Fonte: Autores.

Tabela 7. Tipos de parto dos pacientes.

\begin{tabular}{cccc}
\multicolumn{1}{c}{ Tipo de Parto } & $\mathbf{N}^{\mathbf{0}}$ de Casos & \% \\
& NORMAL & 13 & $56,5 \%$ \\
CESAREO & & 4 & $17,4 \%$ \\
Total & & 6 & $26,1 \%$
\end{tabular}

Fonte: Autores.

Tabela 8. Presença de lesões nas mães dos pacientes.

$\begin{array}{|ccc|}\text { Presença de lesões nas mães } & \mathbf{N}^{\mathbf{0}} \text { de Casos } & \mathbf{\%} \\ \text { SIM } & 12 & 52,2 \% \\ \text { NÃO } & 5 & 21,7 \% \\ \text { DESCONHECIDO } & 6 & 26,1 \% \\ \text { Total } & \mathbf{2 3} & \mathbf{1 0 0 , 0 \%}\end{array}$

Fonte: Autores.

A terapêutica básica instituída foi a de uso exclusivo de CAF em 13 pacientes, o que representou 56,5\% dos tratamentos. CAF associado a IMIQUIMODE, ocorreu em 5 casos, e associado a ATA e IMIQUIMODE em 2 casos, houve 3 casos de remissão espontânea (Tabela 9). Os tratamentos tiveram variação de 0 a 6 sessões, onde a faixa de 0 a 2 sessões representaram 73,9\%. (Figura 10).

Tabela 9. Tipos de tratamento em crianças portadoras de condiloma acuminado.

$\begin{array}{ccc}\text { Tratamento } & \mathbf{N}^{\mathbf{0}} \text { de Casos } & \text { \% } \\ \text { CAF/IMIQUIMODE } & 5 & 21,7 \% \\ \text { CAF } & 13 & 56,5 \% \\ \text { IMIQUIMODE/ CAF/ ATA } & 2 & 8,7 \% \\ \text { REMISSÃO EXPONTANEA } & 3 & 13,0 \% \\ \text { Total } & \mathbf{2 3} & \mathbf{1 0 0 , 0 \%}\end{array}$

Fonte: Autores.

Tabela 10. Quantidade de sessões em crianças portadoras de condiloma acuminado.

$\begin{array}{|ccc|}\text { Quantidade de sessões do tratamento } & \mathbf{N}^{\mathbf{0}} \text { de Casos } & \text { \% } \\ 0-2 & 17 & 73,9 \% \\ 3-4 & 3 & 13,0 \% \\ 5-6 & 3 & 13,0 \% \\ \text { Total } & \mathbf{2 3} & \mathbf{1 0 0 , 0 \%}\end{array}$

Fonte: Autores. 


\section{Discussão}

O HPV pode infectar tanto pele como mucosas. A maioria das infecções regride espontaneamente dentro de dois anos em pessoas com imunidade preservada. Mais de 130 subtipos de HPV são identificados até o momento, embora apenas cerca de um terço deles possa infectar a mucosa anogenital, oral, ou laríngea, predominando os subtipos 6, 11 e 16 (Cherry et al., 2013). A infecção pelo HPV é uma das DST mais comuns em adultos sexualmente ativos (Carr \& Gyorfi, 2000). Aproximadamente $10 \%$ da população adulta apresenta lesões genitais clínicas induzidas pelo vírus (Siegfried et al., 1998). Observamos em nossos resultados que a incidência de abuso sexual, no entanto, foi baixa sendo evidenciada em apenas $8.7 \%$.

A transmissão vertical comumente ocorre durante o parto vaginal em mulheres portadoras do HPV, mesmo que não existam sinais clínicos da doença. Menos comum, a contaminação do recém-nascido em mulheres que realizam a cesariana, parece independer da integridade das membranas, possivelmente por via ascendente transplacentária (Rogo \& Nyansera, 1989). Dados estes que coincidem com nossos achados uma vez que a provável transmissão vertical foi observada em $65,2 \%$. No trabalho de Obalek et al. (1993), no qual se avaliaram crianças com idade entre 7 meses e 12 anos portadoras de verrugas anogenitais, foi constatado uma prevalência de 17,4\% de HPV 2 (causadores da verruga comum) e 74\% de HPV 6. Este trabalho enfatiza que a auto-inoculação por parte de crianças portadoras de verrugas em mãos, ou heteroinoculação pode ser um modo de transmissão não-sexual (Obalek et al., 1993; Yell et al., 1993). A possibilidade de transmissão do HPV para o feto permanece pouco esclarecida, embora existam inferências de que possa ocorrer por via hematogênica, pelo sêmen durante a fertilização, ou de forma ascendente materna, a exemplo do que ocorre em outras infecções durante a gravidez (Syrjanen \& Puranen, 2000).

O local mais comum do aparecimento do condiloma acuminado em crianças é a região perianal, podendo se estender até o canal anal. Particularmente nas meninas, pode-se encontrar lesões periuretrais, himenais e em fúrcula vaginal se apresentando de forma irregular ou como múltiplas pequenas pápulas, estendendo-se até grandes e pequenos lábios (Emans et al., 2005). Segundo Rehme et al. (1998), o local predominante foi vulva e/ou períneo, totalizando 95\% dos achados. Em razão das limitações do exame ginecológico em meninas pré-puberes, o condiloma vaginal e cervical é raramente descrito em literatura. Observamos que em nossa casuística, o local predominante foi vulva e/ou períneo, totalizando 100\% dos achados.

Dentre as manifestações clínicas causadas pela infecção pelo HPV, destacam-se as verrugas cutâneas ou mucosas de localização anogenital, em sua maioria assintomática, que têm sido descritas desde a antiguidade (Robert Snoeck, 2006). Estes dados coincidem com nossos achados, uma vez que a presença de verrugas assintomáticas foi observada em $100 \%$ das pacientes.

$\mathrm{O}$ tratamento direciona-se inicialmente às lesões causadas por HPV, baseando-se em exérese da lesão, métodos citodestrutivos (físicos ou químicos), imunomodulação ou combinação dessas modalidades terapêuticas (Fox \& Tung, 2005; Snoeck et al., 1998). A ablação cirúrgica da lesão mantém-se como a abordagem básica para o tratamento da maioria das lesões anogenitais causadas por HPV. A remoção cirúrgica oferece a vantagem da rápida eliminação da lesão, porém com os inconvenientes associados a qualquer procedimento cirúrgico, como sangramentos e infecção na ferida (Severson et al., 2001). A persistência de lesões induzidas pelo HPV depende principalmente de defeito na imunovigilância(Tchernev, 2009)

\section{Conclusão}

O condiloma acuminado genital foi mais frequente em meninas, esteve associado a provável transmissão vertical em $56,5 \%$ dos casos e apresentou boa resposta terapêutica de um modo geral. A amostra não permite conclusões maiores em relação a via de parto, sendo possível a realização de futuros trabalhos para a avaliação.

\section{Referências}

Armstrong, D. K. B., \& Handley, J. M. (1997). Anogenital warts in prepubertal children: pathogenesis, HPV typing and management. International Journal of $S T D \& A I D S, 8(2), 78-81$. 
Bechtel, K. (2010). Sexual abuse and sexually transmitted infections in children and adolescents. Current Opinion in Pediatrics, 22(1), 94-99. https://doi.org/10.1097/MOP.0b013e32833502ad

Carr, J., \& Gyorfi, T. (2000). Human Papillomavirus: Epidemiology, Transmission, and Pathogenesis. Clinics in Laboratory Medicine, 20(2), 235-255. https://doi.org/10.1016/S0272-2712(18)30060-X

Cherry, J., Demmler-Harrison, G. J., Kaplan, S. L., Steinbach, W. J., \& Hotez, P. J. (2013). Feigin and Cherry's Textbook of Pediatric Infectious Diseases EBook: 2-Volume Set. Elsevier Health Sciences.

Emans, S. J. H., Laufer, M. R., \& Goldstein, D. P. (2005). Pediatric and adolescent gynecology.

Fox, P. A., \& Tung, M.-Y. (2005). Human Papillomavirus. American Journal of Clinical Dermatology, 6(6), 365-381.

Hammerschlag, M. R., \& Guillén, C. D. (2010). Medical and Legal Implications of Testing for Sexually Transmitted Infections in Children. Clinical Microbiology Reviews, 23(3), 493-506. https://doi.org/10.1128/CMR.00024-09

Moresi, J. M., Herbert, C. R., \& Cohen, B. A. (2001). Treatment of Anogenital Warts in Children with Topical 0.05\% Podofilox Gel and 5\% Imiquimod Cream. Pediatric Dermatology, 18(5), 448-450. https://doi.org/10.1046/j.1525-1470.2001.1980a.x

Obalek, S., Misiewicz, J., Jablonska, S., Favre, M., \& Orth, G. (1993). Childhood Condyloma Acuminatum: Association with Genital and Cutaneous Human Papillomaviruses. Pediatric Dermatology, 10(2), 101-106. https://doi.org/10.1111/j.1525-1470.1993.tb00031.x

Rehme, M. F. B., Carvalho, N. S., Ihlenfeld, M. F. K., \& Chuery, A. C. S. (1998). Condiloma Acuminado em Crianças e Adolescentes. Revista Brasileira de Ginecologia e Obstetrícia, 20(7), 377-380. https://doi.org/10.1590/S0100-72031998000700002

Rogo, K. O., \& Nyansera, P. N. (1989). Congenital condylomata acuminata with meconium staining of amniotic fluid and fetal hydrocephalus: case report. East African Medical Journal, 66(6), 411—413. http://europepmc.org/abstract/MED/2791948

Severson, J., Evans, T. Y., Lee, P., Chan, T., Arany, I., \& Tyring, S. K. (2001). Human papillomavirus infections: epidemiology, pathogenesis, and therapy. Journal of Cutaneous Medicine and Surgery: Incorporating Medical and Surgical Dermatology, 5(1), 43-60.

Siegfried, E., Rasnick-Conley, J., Cook, S., Leonardi, C., \& Monteleone, J. (1998). Human Papillomavirus Screening in Pediatric Victims of Sexual Abuse. Pediatrics, 101(1), 43-47. https://doi.org/10.1542/peds.101.1.43

Snoeck, R., Andrei, G., \& Clercq, E. (1998). Specific therapies for human papilloma virus infections. Current Opinion in Infectious Diseases, 11(6), 733-738. https://doi.org/10.1097/00001432-199812000-00014

Snoeck, Robert. (2006). Papillomavirus and treatment. Antiviral Research, 71(2-3), 181-191. https://doi.org/10.1016/j.antiviral.2006.06.007

Syrjanen, S., \& Puranen, M. (2000). Human Papillomavirus Infections in Children: the Potential Role of Maternal Transmission. Critical Reviews in Oral Biology \& Medicine, 11(2), 259-274. https://doi.org/10.1177/10454411000110020801

Tchernev, G. (2009). Sexually transmitted papillomavirus infections: epidemiology pathogenesis, clinic, morphology, important differential diagnostic aspects, current diagnostic and treatment options. Anais Brasileiros de Dermatologia, 84(4), 377-389. https://doi.org/10.1590/S0365-05962009000400009

Unger, E. R., Fajman, N. N., Maloney, E. M., Onyekwuluje, J., Swan, D. C., Howard, L., Beck-Sague, C. M., Sawyer, M. K., Girardet, R. G., Sautter, R. L., Hammerschlag, M. R., \& Black, C. M. (2011). Anogenital Human Papillomavirus in Sexually Abused and Nonabused Children: A Multicenter Study. Pediatrics, 128(3), e658-e665. https://doi.org/10.1542/peds.2010-2247

Yell, J. A., Sinclair, R., Mann, S., Fleming, K., \& Ryan, T. J. (1993). Human papillomavirus type 6-induced condylomata: an unusual complication of intertrigo. British Journal of Dermatology, 128(5), 575-577. https://doi.org/10.1111/j.1365-2133.1993.tb00239.x 